\title{
Vertex stress related parameters for certain Kneser graphs
}

\author{
Johan KOK \\ Independent Mathematics Researcher, City of \\ Tshwane, South Africa \& Visiting Faculty at \\ CHRIST (Deemed to be a University), Bangalore, \\ India \\ email: jacotype@gmail.com \& \\ johan.kok@christuniversity.in
}

\begin{abstract}
This paper presents results for some vertex stress related parameters in respect of specific subfamilies of Kneser graphs. Kneser graphs for which $\operatorname{diam}(K G(n, k))=2$ and $k \geq 2$ are considered. The note establishes the foundation for researching similar results for Kneser graphs for which $\operatorname{diam}(K G(n, k)) \geq 3$. In addition some important vertex stress related properties are stated. Finally some results for specific bipartite Kneser graphs i.e. $B K(n, 1), n \geq 3$ will be presented. In the conclusion some worthy research avenues are proposed.
\end{abstract}

\section{Introduction}

It is assumed that the reader has good working knowledge of set theory. For the general notation, notions and important introductory results in set theory, see [3]. For the general notation, notions and important introductory results in graph theory, see $[2,4]$.

Only non-trivial, finite, undirected and connected simple graphs are considered. Let $X_{i}, i=1,2,3, \ldots,\left(\begin{array}{l}n \\ k\end{array}\right), k \geq 1$ be the $k$-element subsets of the set,

Computing Classification System 1998: G.2.2

Mathematics Subject Classification 2010: 05C12, 05C30, 05 C69

Key words and phrases: vertex stress, diameter, distance, Kneser graph. 
$\{1,2,3, \ldots, n\}$. A Kneser graph denoted by $\operatorname{KG}(n, k), n, k \in \mathbb{N}$ is a graph with vertex set,

and the edge set,

$$
\mathrm{V}(\mathrm{KG}(\mathrm{n}, \mathrm{k}))=\left\{v_{\mathrm{i}}: v_{\mathrm{i}} \mapsto \mathrm{X}_{\mathrm{i}}\right\}
$$

$$
\mathrm{E}(\mathrm{KG}(\mathrm{n}, \mathrm{k}))=\left\{v_{i} v_{j}: X_{i} \cap X_{j}=\emptyset\right\}
$$

Without any relation between $n$ and $k$ the following subfamilies of Kneser graphs follow easily.

1. For $k>n$ the Kneser graph has an empty vertex set implying that the edge set is empty. Hence, for $n \in \mathbb{N}$ the empty graph is obtained.

2 . For $k=1$ the Kneser graph $K G(n, k) \cong K_{n} \forall n$.

3 . For $k=n$ the Kneser graph is always a trivial graph (i.e. $K_{1}$ ).

4. For $\frac{n}{2}<k<n$ the vertex set is non-empty. However, the edge set is empty so for the permissible values of $k$ and $n$ the corresponding null graphs $\mathfrak{N}_{t}$, $\mathrm{t}=\left(\begin{array}{l}\mathrm{n} \\ \mathrm{k}\end{array}\right)$ are obtained.

5 . For $n$ even and $k=\frac{n}{2}$ a corresponding matching graph is obtained.

6. For $n$ even and $2 \leq k \leq \frac{n-2}{2}$ and; for $n$ odd and $2 \leq k \leq\left\lfloor\frac{n}{2}\right\rfloor$ the subfamily of non-trivial, connected and non-complete Kneser graphs is obtained.

Note that the author draws a distinction between an empty graph $\mathrm{G}$, (both $\mathrm{V}(\mathrm{G})=\emptyset$ and $\mathrm{E}(\mathrm{G})=\emptyset)$ and a null graph $\mathrm{H},(\mathrm{V}(\mathrm{H}) \neq \emptyset$ and $\mathrm{E}(\mathrm{H})=\emptyset)$. This distinction is not common in the literature. Conventionally, the 0-element subset is not considered. However, from set theory it is known that the emptyset is indeed a subset of any set. Therefore, it seems proper to state that $\mathrm{KG}(\mathrm{n}, 0)$ is a trivial graph say, $v_{1} \mapsto \emptyset$.

It follows directly from the structure of a Kneser graph that the order of a Kneser graph is given by $v(K G(n, k))=\left(\begin{array}{l}n \\ k\end{array}\right)$. Furthermore, because vertex adjacency as it relates to a k-element subset is defined without loss of generality, a Kneser graph is inherently a degree regular graph. The number of neighbors of any vertex $v_{i}$ is given by, $\operatorname{deg}\left(v_{i}\right)=\left(\begin{array}{c}n-k \\ k\end{array}\right)$. From the aforesaid it follows that the number of edges is given by, $\varepsilon(K G(n, k))=\frac{1}{2} \times \frac{n !}{k ! k !(n-2 k) !}$.

Example: $\mathrm{KG}(5,2)$ : Let $\mathrm{V}(\mathrm{KG}(5,2))$ be define as: $v_{1} \mapsto\{1,2\}, v_{2} \mapsto\{1,3\}$, $v_{3} \mapsto\{1,4\}, v_{4} \mapsto\{1,5\}, v_{5} \mapsto\{2,3\}, v_{6} \mapsto\{2,4\}, v_{7} \mapsto\{2,5\}, v_{8} \mapsto\{3,4\}, v_{9} \mapsto$ $\{3,5\}, v_{10} \mapsto\{4,5\}$. See figure 1 as illustration. It is known that, $\operatorname{KG}(5,2) \cong$ Petersen graph. 


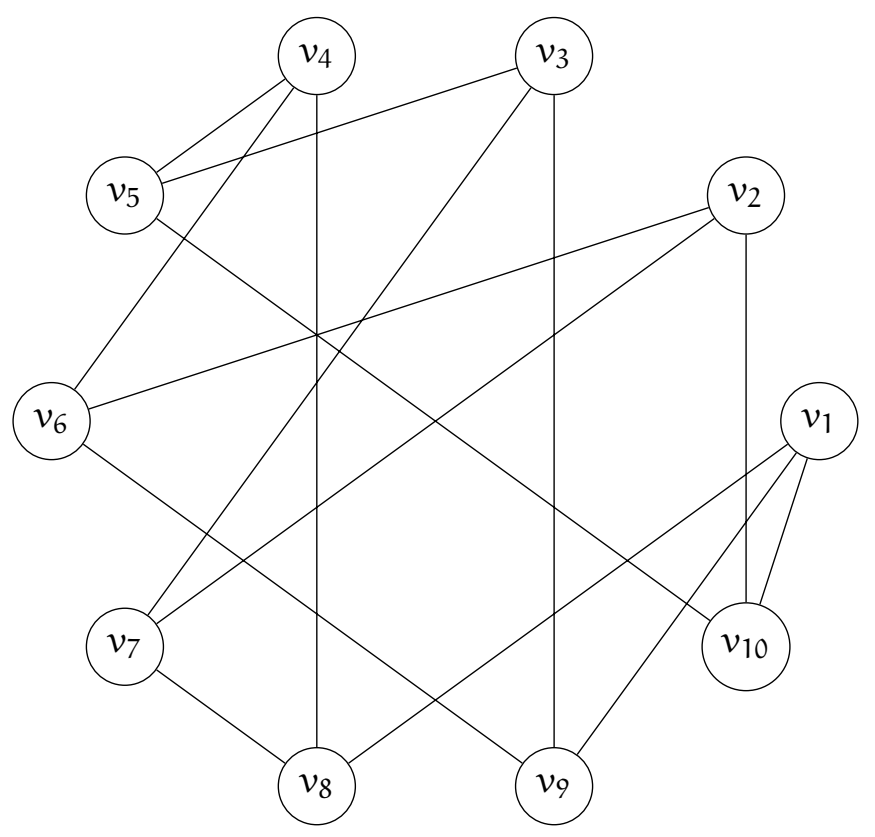

Figure 1: KG(5,2) of order 10.

\section{Total induced vertex stress, total vertex stress and vertex stress}

The vertex stress of vertex $v \in \mathrm{V}(\mathrm{G})$ is the number of times $v$ is contained as an internal vertex in all shortest paths between all pairs of distinct vertices in $\mathrm{V}(\mathrm{G}) \backslash\{v\}$. Formally stated, $\mathcal{S}_{\mathrm{G}}(v)=\sum_{\mathfrak{u} \neq w \neq v \neq u} \sigma(v)$ with $\sigma(v)$ the number of shortest paths between vertices $u, w$ which contain $v$ as an internal vertex. Such a shortest $u \mathfrak{w}$-path is also called a $u \mathfrak{w}$-distance path. See [8, 9]. The total vertex stress of $\mathrm{G}$ is given by $\mathcal{S}(\mathrm{G})=\sum_{v \in V(\mathrm{G})} \mathcal{S}_{\mathrm{G}}(v)$, [5]. From [10, 11] we recall the definition of total induced vertex stress denoted by, $\mathfrak{s}_{\mathrm{G}}\left(v_{\mathrm{i}}\right), v_{\mathrm{i}} \in \mathrm{V}(\mathrm{G})$.

Definition 1 [11] Let $\mathrm{V}(\mathrm{G})=\left\{v_{\mathrm{i}}: 1 \leq \boldsymbol{i} \leq \mathrm{n}\right\}$. For the ordered vertex pair $\left(v_{i}, v_{j}\right)$ let there be $\mathrm{k}_{\mathrm{G}}(i, j)$ distinct shortest paths of length $\mathrm{l}_{\mathrm{G}}(i, j)$ from $v_{i}$ to $v_{j}$. Then, $\mathfrak{s}_{G}\left(v_{i}\right)=\sum_{j=1, j \neq i}^{n} k_{G}(i, j)\left(\ell_{G}(i, j)-1\right)$.

The notion of vertex stress finds application in research related to centrality in graphs. In dynamical graph theory the parameter assists to identify vertices 
which are more prone to system failure. The nodes within road networks are a good example. A more subtle example is identifying the possibility of a high number of step-through certain steps in an algorithm. Such high number step-through may lead to excessive memory requirements. Highly congested airports can be pre-empted by determining the vertex stress of airports serving as the nodes of a flight route network.

The families no. 1 to 5 have the vertex stress related parameters equal to 0 . The only interesting family of Kneser graphs to consider with regards to vertex stress and related parameters is family no. 6. Thus only Kneser graphs within the range $2 \leq k<\frac{n}{2}$ will be studied. From [12] we have that $\operatorname{diam}(K G(n, k))=1+\left\lceil\frac{k-1}{n-2 k}\right\rceil$. Hence, for $2 \leq k \leq \frac{n+1}{3}$ a corresponding Kneser $\operatorname{graph} \operatorname{has} \operatorname{diam}(K G(n, k))=2$. This section seeks to find results for $k \geq 2$ subject to, $n \geq 3 k-1$.

Case 1: Let $k=2$, then $n \geq 5$. For ease of reasoning the following convention for 2 -subsets of the set $\{1,2,3, \ldots, n\}$ will be used. The vertices are defined as: $v_{1} \mapsto\{1,2\}, v_{2} \mapsto\{1,3\}, \ldots, v_{n-1} \mapsto\{1, n\}, v_{n} \mapsto\{2,3\}, v_{n+1} \mapsto$ $\{2,4\}, \ldots, v_{2 n-3} \mapsto\{2, n\}, \ldots, v_{\left(\begin{array}{c}n \\ 2\end{array}\right)} \mapsto\{n-1, n\}$.

Remark. As stated before, since vertex adjacency is defined without loss of generality (for brevity, the $w$ lg-principle) all results in respect of vertex $v_{1}$ are (immediately) valid for all $v_{i} \in \mathrm{V}(\mathrm{KG}(\mathrm{n}, \mathrm{k}))$. Hence, for ease of reasoning the results for $v_{1}$ will be determined and then generalized. Such generalization is axiomatically valid and requires no further proof.

Recall that the set of vertices adjacent to vertex $v_{i}$ is called the open neighborhood of $v_{i}$ and it is denoted by $\mathrm{N}\left(v_{i}\right)$ (or $\mathrm{N}_{\mathrm{G}}\left(v_{i}\right)$ if reference to $\mathrm{G}$ is important). The closed neighborhood of vertex $v_{i}$ is defined as, $N\left[v_{i}\right]=N\left(v_{i}\right) \cup\left\{v_{i}\right\}$ (or $\mathrm{N}_{\mathrm{G}}\left[v_{i}\right]$ if reference to $\mathrm{G}$ is important).

Proposition 2 For a Kneser graph $\mathrm{KG}(\mathrm{n}, 2), \mathrm{n} \geq 5$ the total induced vertex stress of $v_{1}$ is given by

$$
\mathfrak{s}_{\mathrm{KG}(\mathrm{n}, 2)}\left(v_{1}\right)=\left[\left(\begin{array}{c}
\mathrm{n} \\
2
\end{array}\right)-\left(\left(\begin{array}{c}
\mathrm{n}-2 \\
2
\end{array}\right)+1\right)\right] \times\left(\begin{array}{c}
\mathrm{n}-3 \\
2
\end{array}\right) .
$$

Proof. Clearly, $\mathrm{N}\left(v_{1}\right)=\left\{v_{i}: 1,2 \notin v_{i}\right\}$. It is known that $\left|\mathrm{N}\left(v_{1}\right)\right|=\left(\begin{array}{c}\mathrm{n}-2 \\ 2\end{array}\right)$. $\operatorname{Because} \operatorname{diam}(K G(n, k))=2$ there does not exist any shortest 3-path in $\mathrm{KG}(\mathrm{n}, 2)$. Hence, there only exist shortest 2-paths from $v_{1}$ to the remaining $\left(\begin{array}{c}n \\ 2\end{array}\right)-\left(\left(\begin{array}{c}n-2 \\ 2\end{array}\right)+1\right)$ vertices of $K G(n, 2)$. Without loss of generality consider vertex $v_{2} \mapsto\{1,3\}$. Since $\mathbf{N}\left(v_{2}\right)=\left\{v_{j}: 1,3 \notin v_{j}\right\}$ it follows easily that $\left|\mathbf{N}\left(v_{1}\right) \cap \mathbf{N}\left(v_{2}\right)\right|=$ $\left(\begin{array}{c}n-3 \\ 2\end{array}\right)$. The aforesaid is true because the elements $1,2,3$ are (must be) excluded 
as elements of the 2-element subsets in $\mathrm{N}\left(v_{1}\right) \cap \mathrm{N}\left(v_{2}\right)$. Thus, the partial total vertex stress induced by vertex $v_{1}$ along all shortest $v_{1} v_{2}$-paths is settled. By the $w \mathrm{lg}$-principle the principle of immediate induction is valid. Therefore,

$$
\mathfrak{s}_{\mathrm{KG}(\mathrm{n}, 2)}\left(v_{1}\right)=\left[\left(\begin{array}{c}
\mathrm{n} \\
2
\end{array}\right)-\left(\left(\begin{array}{c}
\mathrm{n}-2 \\
2
\end{array}\right)+1\right)\right] \times\left(\begin{array}{c}
\mathrm{n}-3 \\
2
\end{array}\right) .
$$

The generalized corollaries follow immediately.

Corollary 3 For a Kneser graph $\mathrm{KG}(\mathrm{n}, 2), \mathrm{n} \geq 5$ the total induced vertex stress of $v_{i} \in \mathrm{V}(\mathrm{KG}(\mathrm{n}, 2))$ is given by,

$$
\mathfrak{s}_{K G(n, 2)}\left(v_{i}\right)=\left[\left(\begin{array}{c}
n \\
2
\end{array}\right)-\left(\left(\begin{array}{c}
n-2 \\
2
\end{array}\right)+1\right)\right] \times\left(\begin{array}{c}
n-3 \\
2
\end{array}\right) .
$$

Corollary 4 For a Kneser graph $\mathrm{KG}(\mathrm{n}, 2), \mathrm{n} \geq 5$ the total vertex stress is given by

$$
\mathcal{S}(\mathrm{KG}(\mathrm{n}, 2))=\frac{1}{2}\left(\begin{array}{c}
\mathrm{n} \\
2
\end{array}\right)\left[\left(\begin{array}{c}
\mathrm{n} \\
2
\end{array}\right)-\left(\left(\begin{array}{c}
\mathrm{n}-2 \\
2
\end{array}\right)+1\right)\right] \times\left(\begin{array}{c}
\mathrm{n}-3 \\
2
\end{array}\right) .
$$

Proof. The result follows from Definition 1 read together with the proof of Proposition 2 and Corollary 3.

Corollary 5 For a Kneser graph $\mathrm{KG}(\mathrm{n}, 2), \mathrm{n} \geq 5$ the vertex stress is given by

$$
\mathcal{S}_{\mathrm{KG}(\mathrm{n}, 2)}\left(v_{i}\right)=\frac{1}{2}\left[\left(\begin{array}{c}
n \\
2
\end{array}\right)-\left(\left(\begin{array}{c}
n-2 \\
2
\end{array}\right)+1\right)\right] \times\left(\begin{array}{c}
n-3 \\
2
\end{array}\right) .
$$

Proof. It is known that the Kneser graphs $K G(n, 2)$ are distance regular, see [1]. By Theorem 3.6 in [9] the Kneser graphs $\mathrm{KG}(n, 2)$ are stress regular as well. Thus, the result of Corollary 4 must simply be divided by $\left(\begin{array}{c}\mathfrak{n} \\ 2\end{array}\right)$.

Note that since $2 \leq k \leq \frac{n+1}{3}$ it follows that $n \geq 3 k_{1}-1$ for a $k_{1} \in \mathbb{N} \backslash\{1\}$ to ensure that $\operatorname{diam}\left(K G\left(n, k_{1}\right)\right)=2$. This observation enables immediate generalizations. The vertices which may be used for reasoning of proof are:

$$
\begin{gathered}
v_{1} \mapsto\left\{1,2,3, \ldots, \mathrm{k}_{1}-1, \mathrm{k}_{1}\right\}, v_{2} \mapsto\left\{1,2,3, \ldots, \mathrm{k}_{1}-1, \mathrm{k}_{1}+1\right\} \\
\text { and } v_{\mathrm{i}} \in \mathrm{N}\left(v_{1}\right) \cap \mathrm{N}\left(v_{2}\right) .
\end{gathered}
$$

Because the reasoning of proof is similar to that found in Proposition 2 and Corollaries 3 to 5 and the fact that the $w \mathrm{lg}$-principle applies throughout in all Kneser graph embodiments, no further proofs are presented.

Theorem 6 For a Kneser graph $\mathrm{KG}\left(\mathrm{n}, \mathrm{k}_{1}\right), \mathrm{k}_{1} \in \mathbb{N} \backslash\{1,2\}, \mathrm{n} \geq 3 \mathrm{k}_{1}-1$ the total induced vertex stress of $v_{\mathrm{i}} \in \mathrm{V}\left(\mathrm{KG}\left(\mathrm{n}, \mathrm{k}_{1}\right)\right)$ is given by

$$
\mathfrak{s}_{K G\left(n, k_{1}\right)}\left(v_{i}\right)=\left[\left(\begin{array}{c}
n \\
k_{1}
\end{array}\right)-\left(\left(\begin{array}{c}
n-k_{1} \\
k_{1}
\end{array}\right)+1\right)\right] \times\left(\begin{array}{c}
n-\left(k_{1}+1\right) \\
k_{1}
\end{array}\right) .
$$


Corollary 7 For a Kneser graph $\mathrm{KG}\left(\mathrm{n}, \mathrm{k}_{1}\right), \mathrm{k}_{1} \in \mathbb{N} \backslash\{1,2\}, \mathrm{n} \geq 3 \mathrm{k}_{1}-1$ the total vertex stress is given by

$$
\mathcal{S}\left(K G\left(n, k_{1}\right)\right)=\frac{1}{2}\left(\begin{array}{c}
n \\
k_{1}
\end{array}\right)\left[\left(\begin{array}{c}
n \\
k_{1}
\end{array}\right)-\left(\left(\begin{array}{c}
n-k_{1} \\
k_{1}
\end{array}\right)+1\right)\right] \times\left(\begin{array}{c}
n-\left(k_{1}+1\right) \\
k_{1}
\end{array}\right) .
$$

Corollary 8 For a Kneser graph $\mathrm{KG}\left(\mathrm{n}, \mathrm{k}_{1}\right), \mathrm{k}_{1} \in \mathbb{N} \backslash\{1,2\}, \mathrm{n} \geq 3 \mathrm{k}_{1}-1$ the vertex stress is given by,

$$
\mathcal{S}_{\mathrm{KG}\left(n, k_{1}\right)}\left(v_{i}\right)=\frac{1}{2}\left[\left(\begin{array}{c}
n \\
k_{1}
\end{array}\right)-\left(\left(\begin{array}{c}
n-k_{1} \\
k_{1}
\end{array}\right)+1\right)\right] \times\left(\begin{array}{c}
n-\left(k_{1}+1\right) \\
k_{1}
\end{array}\right) .
$$

\subsection{Vertex stress related properties of $K G(n, 2)$}

Recall some results from [9]. A graph $\mathrm{G}$ for which $\mathcal{S}_{\mathrm{G}}\left(v_{\mathrm{i}}\right)=\mathcal{S}_{\mathrm{G}}\left(v_{j}\right)$ for all distinct pairs $v_{i}, v_{j} \in \mathrm{V}(\mathrm{G})$ is said to be stress regular.

Theorem 9 [9] Every distance regular graph is stress regular.

Corollary 10 [9] Every strongly regular graph is stress regular.

Corollary 11 [9] Every distance transitive graph is stress regular.

Since it is known that the family of Kneser graphs $K G(n, 2)$ are distance regular graphs it follows from Theorem 9 that the Kneser graphs KG(n, 2) are stress regular. Furthermore, it is known from [1] that every distance regular graph $\mathrm{G}$ with $\operatorname{diam}(\mathrm{G})=2$, is strongly regular. The aforesaid read together with Corollary 10 permit the next corollary without further proof.

Corollary 12 Kneser graphs $\mathrm{KG}\left(\mathrm{n}, \mathrm{k}_{1}\right), \mathrm{k}_{1} \in \mathbb{N} \backslash\{1,2\}, \mathrm{n} \geq 3 \mathrm{k}_{1}-1$ are stress regular.

In fact, a general result (without further proof) is permitted from the knowledge that all Kneser graphs $K G(n, k), n \geq k$ are vertex transitive.

Theorem 13 All Kneser graphs $\mathrm{KG}(\mathrm{n}, \mathrm{k}), \mathrm{n} \geq \mathrm{k}$ are stress regular.

\subsection{Stress balanced graphs}

Definition 14 A graph $\mathrm{G}$ is said to be stress-balanced if and only if

$$
\sum_{v_{\mathrm{t}} \in \mathrm{N}\left[v_{\mathrm{i}}\right]} \mathcal{S}_{\mathrm{G}}\left(v_{\mathrm{t}}\right)=\sum_{v_{\mathrm{l}} \in \mathrm{N}\left[v_{\mathrm{j}}\right]} \mathcal{S}_{\mathrm{G}}\left(v_{\mathrm{l}}\right)
$$

for all pairs of distinct vertices $v_{i}, v_{j} \in \mathrm{V}(\mathrm{G})$. 
The value $\gamma\left(v_{i}\right)=\sum_{v_{t} \in \mathrm{N}\left[v_{i}\right]} \mathcal{S}_{\mathrm{G}}\left(v_{\mathrm{t}}\right)$ is called the vertex stress index of the vertex $v_{i}$. A star graph (for brevity, a star) is a tree which has a central vertex $v_{0}$ with $m \geq 0$ pendent vertices (or leafs) attached to $v_{0}$. The star is denoted by $S_{1, m}$ and the leafs are labeled, $v_{i}, i=1,2,3, \ldots, m$. It is straightforward to verify that the respective vertex stress are, $\mathcal{S}_{\mathrm{S}_{1, \mathrm{~m}}}\left(\nu_{\mathrm{i}}\right)=0,1 \leq i \leq \mathrm{m}$ and $\mathcal{S}_{\mathrm{S}_{1, \mathrm{~m}}}\left(v_{0}\right)=\frac{\mathrm{m}(\mathrm{m}-1)}{2}$. Since, $\sum_{v_{\mathrm{j}} \in \mathrm{N}_{\mathrm{S}_{1, \mathrm{~m}}}\left[v_{0}\right]} \mathcal{S}_{\mathrm{S}_{1, \mathrm{~m}}}\left(v_{\mathrm{j}}\right)=\frac{\mathrm{m}(\mathrm{m}-1)}{2}+\mathrm{m} \times 0=\frac{\mathrm{m}(\mathrm{m}-1)}{2}$ and $\sum_{v_{j} \in N_{S_{1, m}}\left[v_{i}\right]} \mathcal{S}_{S_{1, m}}\left(v_{j}\right)=0+\frac{\mathfrak{m}(m-1)}{2}=\frac{m(m-1)}{2}, 1 \leq i \leq m$ a star is stressbalanced. A star shows that, despite not being degree regular or stress regular, a star is stress-balanced. Figure 2 depicts another example. The graph $\mathrm{G}=$ $\mathrm{C}_{4}+v_{1} v_{3}$ is not degree regular and has $\mathcal{S}_{\mathrm{G}}\left(v_{1}\right)=\mathcal{S}_{\mathrm{G}}\left(v_{3}\right)=1$ and $\mathcal{S}_{\mathrm{G}}\left(v_{2}\right)=$ $\mathcal{S}_{\mathrm{G}}\left(v_{4}\right)=0$. So $\mathrm{G}$ is not stress regular but it is stress-balanced.

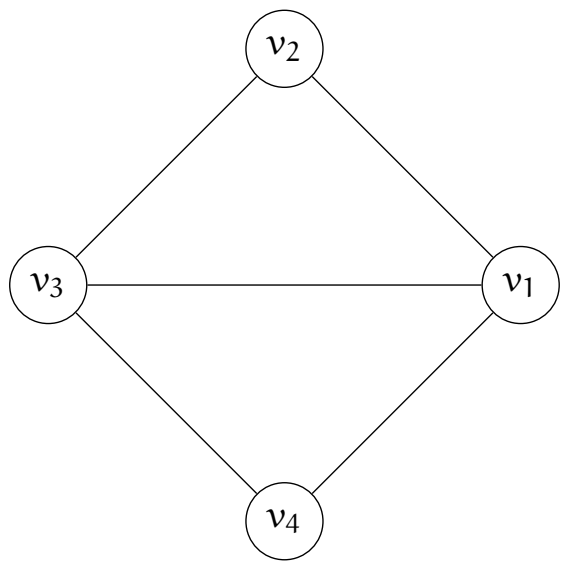

Figure 2: $\mathrm{G}=\mathrm{C}_{4}+v_{1} v_{3}$.

Lemma 15 A graph $\mathrm{G}$ which is degree regular (or regular for brevity) and stress regular is stress-balanced.

Proof. The result is a direct consequence of Definition 14 .

We present the main result of this subsection.

Theorem 16 All Kneser graphs $\mathrm{KG}(\mathrm{n}, \mathrm{k}), \mathrm{n} \geq \mathrm{k}$ are stress-balanced.

Proof. Since all Kneser graphs $K G(n, k), n \geq k$ are degree regular and stress regular (see Theorem 13), read together with Definition 14 and Lemma 15, settles the result. 


\section{On bipartite Kneser graphs, BK(n, k)}

Without loss of generality let $n \geq 3$ and let $1 \leq k \leq\left\lceil\frac{n}{2}\right\rceil-1$. Let $X_{i}, i=$ $1,2,3, \ldots,\left(\begin{array}{l}n \\ k\end{array}\right)$ be the k-element subsets of the set, $\{1,2,3, \ldots, n\}$. Let $Y_{i}, i=$ $1,2,3, \ldots,\left(\begin{array}{l}n \\ k\end{array}\right)$ be the $(n-k)$-element subsets of the set, $\{1,2,3, \ldots, n\}$. Let $\mathrm{V}_{1}=\left\{v_{i}: v_{i} \mapsto X_{i}\right\}$ and $V_{2}=\left\{u_{i}: u_{i} \mapsto Y_{i}\right\}$. A connected bipartite Kneser graph denoted by $B K(n, k)$ is a graph with vertex set,

$$
\mathrm{V}(\mathrm{BG}(\mathrm{n}, \mathrm{k}))=\mathrm{V}_{1} \cup \mathrm{V}_{2}
$$

and the edge set,

$$
\mathrm{E}(\mathrm{BG}(\mathrm{n}, \mathrm{k}))=\left\{v_{i} u_{j}: X_{i} \subset Y_{j}\right\}
$$

From the definition it is axiomatically true (or from applying the $w \mathrm{lg}$-principle) that $\operatorname{BK}(n, k)$ is degree regular with $\operatorname{deg}\left(v_{i}\right)=\operatorname{deg}\left(u_{j}\right)=\left(\begin{array}{c}n-k \\ k\end{array}\right)$. Equally straightforward is that $B K(n, k)$ is of order $2 \times\left(\begin{array}{l}n \\ k\end{array}\right)$. In fact, $\left|V_{1}\right|=\left|V_{2}\right|=\left(\begin{array}{l}n \\ k\end{array}\right)$.

Theorem 17 A bipartite Kneser graph $\mathrm{BK}(\mathrm{n}, \mathrm{k})$ has diam $(\mathrm{BK}(\mathrm{n}, \mathrm{k})) \geq 3$.

Proof. Since $\left(\begin{array}{c}n-k \\ k\end{array}\right)<\left(\begin{array}{l}n \\ k\end{array}\right)$ it follows that $N\left(v_{i}\right) \subset V_{2}$ and similarly, $N\left(u_{j}\right) \subset V_{1}$. Hence, there exists at least one shortest $v_{i} u_{j}$-path (or shortest $u_{j} v_{i}$-path) of distance greater or equal to 3 .

Similar to the notion of stress regularity it is said that a graph $\mathrm{G}$ is induced vertex stress regular (for brevity, IVS-regular) if and only if $\mathfrak{s}_{\mathrm{G}}\left(v_{i}\right)=\mathfrak{s}_{\mathrm{G}}\left(v_{\mathrm{j}}\right)$ for all distinct pairs $v_{i}, v_{j} \in \mathrm{V}(\mathrm{G})$.

Theorem 18 An IVS-regular graph $\mathrm{G}$ is stress regular.

Proof. The result follows from the fact that for any vertex $v_{i}$ the vertex stress is given by, $\mathcal{S}_{\mathrm{G}}\left(v_{\mathrm{i}}\right)=\frac{\mathfrak{s}_{\mathrm{G}}\left(v_{\mathrm{i}}\right)}{2}$.

Corollary 19 An IVS-regular graph G with a singular adjacency regime for all vertices is stress-balanced.

Proof. The result follows from Theorem 18 and the fact that $\operatorname{deg}_{G}\left(v_{i}\right)=$ $\operatorname{deg}_{G}\left(v_{j}\right)$ for all distinct pairs $v_{i}, v_{j} \in V(G)$.

Theorem 18 and Corollary 19 read together with the definition of $B K(n, k)$ imply that bipartite Kneser graphs are stress-balanced. 


\subsection{Specific results for $\operatorname{BK}(n, 1), n \geq 3$}

Theorem 20 Bipartite Kneser graphs, BK( $\mathrm{n}, 1), \mathrm{n} \geq 3$ are stress regular.

Proof. Proposition 3.4 in [7] convinces that a graph $B K(n, 1)$ is distancetransitive. Also, distance-transitive $\Rightarrow$ distance regular. Therefore, the latter read together with Theorem 9 (Theorem 3.6 in [9]) settles the fact that bipartite Kneser graphs $B K(n, 1), n \geq 3$ are stress regular.

Theorem 21 A bipartite Kneser graph, BK(n,1), $\mathrm{n} \geq 3$ has,

$$
\operatorname{diam}(\operatorname{BK}(n, 1))=3 \text {. }
$$

Proof. Let $\mathrm{V}_{1}(\operatorname{BK}(3,1))=\left\{v_{i} \mapsto\{\mathfrak{i}\}: \mathfrak{i}=1,2,3\right.$ and $\mathrm{V}_{2}(\mathrm{BK}(\mathrm{n}, 1))=\left\{\mathrm{u}_{1} \mapsto\right.$ $\left.\{1,2\}, u_{2} \mapsto\{1,3\}, u_{3} \mapsto\{2,3\}\right\}$. From the definition of $B K(n, 1)$ it follows immediately that $\mathrm{BK}(3,1) \cong \mathrm{C}_{6}$. Hence, $\operatorname{diam}(\mathrm{BK}(3,1))=3$. For $\mathrm{B}(4,1)$ each vertex $u_{i} \in V_{2}(B(3,1))$ becomes $u_{i} \cup\{4\}$ and exactly two vertices are added. Therefore, $V_{1}(B K(4,1))=V_{1}(B K(3,1)) \cup\{4\}$ and $V_{2}(B K(4,1))=\left\{u_{i} \cup\{4\}\right.$ : $\left.u_{i} \in V_{2}(B K(3,1))\right\} \cup\{1,2,3\}$. After adding the edges in accordance with the adjacency definition it follows easily that, $\operatorname{diam}(\operatorname{BK}(4,1))=3$. Obviously the vertex changes and the addition of exactly two new vertices remain consistent as $n$ progresses consecutively through $5,6,7 \ldots$

Assume the result holds for $\operatorname{BK}(n, 1), 5 \leq n \leq k$. By similar reasoning to show the result for the progression from $n=3$ to $n=4$, it follows by immediate induction that the results holds for the progression from $n=k$ to $\mathrm{n}=\mathrm{k}+1$. Thus,

$$
\operatorname{BK}(n, 1), n \geq 3 \text { has } \operatorname{diam}(\operatorname{BK}(n, 1))=3 .
$$

Proposition $22 A$ vertex $v_{i} \in \mathrm{V}_{1}(\mathrm{BK}(\mathrm{n}, 1))$ (or $\mathrm{u}_{\mathrm{j}} \in \mathrm{V}_{2}(\mathrm{BK}(\mathrm{n}, 1))$ ) has:

$$
\mathfrak{s}_{\mathrm{BK}(\mathrm{n}, 1)}\left(v_{i}\right)=3 \times \operatorname{deg}_{\mathrm{BK}(\mathrm{n}, 1)}\left(v_{i}\right)\left(\operatorname{deg}_{\mathrm{BK}(\mathrm{n}, 1)}\left(v_{i}\right)-1\right) .
$$

Proof. It follows from Theorem 21 that a vertex $v_{i} \in V_{1}(B K(n, 1))\left(\right.$ or $u_{j} \in$ $\left.\mathrm{V}_{2}(\mathrm{BK}(\mathrm{n}, 1))\right)$ has exactly,

$$
\operatorname{deg}_{B K(n, 1)}\left(v_{i}\right)\left(\operatorname{deg}_{B K(n, 1)}\left(v_{i}\right)-1\right) \text { shortest 2-paths }
$$

and exactly,

$$
\operatorname{deg}_{B K(n, 1)}\left(v_{i}\right)\left(\operatorname{deg}_{B K(n, 1)}\left(v_{i}\right)-1\right) \text { shortest 3-paths. }
$$

Obviously $v_{1}$ has $\operatorname{deg}_{\mathrm{BK}(\mathrm{n}, 1)}\left(v_{\mathrm{i}}\right)$ shortests 1 -paths (or edges). Applying Definition 1 settles the result. 
Corollary 23 (a) A vertex $v_{i} \in \mathrm{V}_{1}(\mathrm{BK}(\mathrm{n}, 1))$ (or $\mathrm{u}_{\mathrm{j}} \in \mathrm{V}_{2}(\mathrm{BK}(\mathrm{n}, 1))$ ) has:

$$
\mathcal{S}_{\mathrm{BK}(\mathrm{n}, 1)}\left(v_{\mathrm{i}}\right)=\frac{3(\mathrm{n}-1)(\mathrm{n}-2)}{2} .
$$

(b) $\operatorname{BK}(n, 1), n \geq 3$ has, $\mathcal{S}(\operatorname{BK}(n, 1))=3 n(n-1)(n-2)$.

(c) $\operatorname{BK}(n, 1), n \geq 3$ has, $\mathfrak{s}(\operatorname{BK}(n, 1))=6 n(n-1)(n-2)$.

Proof. Trivial from the appropriate definitions.

\section{Conclusion}

Author is of the view that an extension of this paper through a study of Kneser graphs with diameter greater than 2 is a worthy endeavor. To ensure that say, $\operatorname{diam}(K G(n, k))=3$ it follows that for $k \geq 3, n \geq \frac{5 k-1}{2}$. The number of vertices (k-subsets) of $\left(\geq \frac{5 k-1}{k^{2}}\right)$ becomes large very rapidly. More so for $\operatorname{diam}(K G(n, k))=\ell, \ell \geq 4$. It is suggested that shortest path algorithms (existing or newly developed) or experimental mathematics through simulation programs be utilized in support of further research.

The new notion of stress-balanced graphs has only been briefly introduced. It is suggested to be an interesting concept with a wide scope for further research. A graph $G$ which is not stress-balanced will have at least one vertex say, $v_{i}$ such that $\gamma\left(v_{i}\right)=\max \left\{\gamma\left(v_{j}\right): \gamma\left(v_{j}\right)=\sum_{v_{\mathrm{t}} \in \mathrm{N}\left[v_{j}\right]} \mathcal{S}_{\mathrm{G}}\left(v_{\mathrm{t}}\right)\right\}$. A closed neighborhood $\mathrm{N}\left[v_{\mathrm{i}}\right]$ which yields such maximum is called a stress district of $\mathrm{G}$. Similarly a closed neighborhood $\mathrm{N}\left[v_{\mathrm{j}}\right]$ which yields $\min \left\{\gamma\left(v_{\mathrm{k}}\right): \gamma\left(v_{\mathrm{k}}\right)=\sum_{v_{\mathrm{t}} \in \mathrm{N}\left[v_{\mathrm{k}}\right]} \mathcal{S}_{\mathrm{G}}\left(\nu_{\mathrm{t}}\right)\right\}$ is called a stress suburb of G. Studying stress districts and stress suburbs remains open.

Various studies of other families of graphs which are constructed from the subsets of a set together with a well-defined adjacency regime have been published. We refer to this as the study of graphs from sets. A specific and perhaps less known family called set-graphs can be read in [6]. Hence, various research projects under the theme Vertex stress related parameters for graphs from sets remain open.

\section{Acknowledgment}

The author would like to thank the anonymous referee for his(her) constructive comments, which helped to improve the elegance and the content enhancement (bipartite Kneser graphs) of this paper. 


\section{References}

[1] N. Biggs, Algebraic Graph Theory $2^{\text {nd }}$ Edition, Cambridge University Press, Cambridge, 1993, ISBN-13: 978-0521458979. $\Rightarrow 328,329$

[2] J.A. Bondy, U.S.R. Murty, Graph Theory with Applications, Macmillan Press, London, $1976 . \Rightarrow 324$

[3] R.C. Freiwald, An Introduction to Set Theory and Topology, Washington State University, Saint. Louis, (2014), http://doi.org/10.7936/K7D798QH $\Rightarrow 324$

[4] F. Harary, Graph Theory, Addison-Wesley, Reading MA, 1969. $\Rightarrow 324$

[5] J. Kok, J. Shiny, V. Ajitha, Total vertex stress alteration in cycle related graphs, Matematichki Bilten, 44, 2 (2020) 149-162. http://doi .org/10.37560/ matbil2020149k $\Rightarrow 326$

[6] J. Kok, K. P. Chithra, N.K. Sudev, C. Susanth, A study on set-graphs, International Journal of Computer Applications, 118, 7, (2015) 1-5. http://doi.org/ 10.5120/20754-3173 $\Rightarrow 333$

[7] S. M. Mirafzal, A. Zafari, Some algebraic properties of bipartite Kneser graphs, arXiv: 1804.04570V1, (2018) 1-11, to appear in Ars Combinatoria. $\Rightarrow 332$

[8] A. Shimbel, Structural parameters of communication networks, The Bulletin of Mathematical Biophysics, 15, 4 (1953) 501-507. $\Rightarrow 326$

[9] J. Shiny, V. Ajitha, Stress regular graphs, Malaya Journal of Matematik, 8, 3 (2020) 1152-1154. http://doi.org/10.26637/MJM0803/0072 $\Rightarrow 326,328,329$, 332

[10] J. Shiny, Induced stress of some graph operations, Malaya Journal of Matematik, 9(1), (2021), 259-261. https://doi.org/10.26637/MJM0901/0043 $\Rightarrow 326$

[11] J. Shiny, J. Kok, V. Ajitha, Total induced vertex stress in barbell-like graphs, Journal of the Indonesian Mathematical Society, 27(2), (2021), 150-157. $\Rightarrow 326$

[12] M. Valencia-Pabon and J-C. Vera, On the diameter of Kneser graphs, Discrete Mathematics, 305 (1-3), (2005), 383-385. $\Rightarrow 327$

Received: November 14, 2021 • Revised: November 27, 2021 